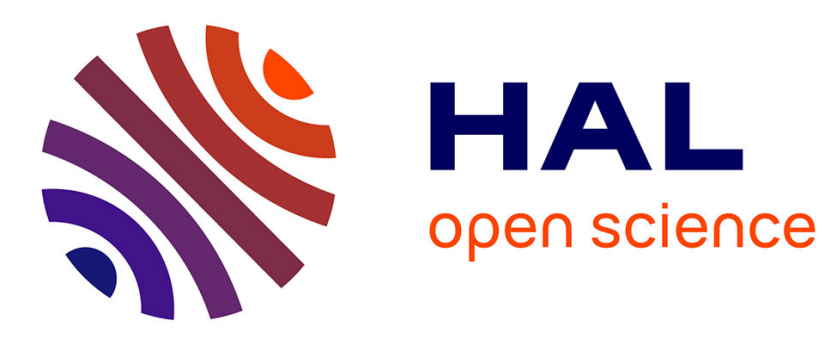

\title{
Stability conditions for some distributed systems : buffered random access systems
}

Wojciec Szpankowski

\section{To cite this version:}

Wojciec Szpankowski. Stability conditions for some distributed systems: buffered random access systems. [Research Report] RR-1815, INRIA. 1992. inria-00074857

\section{HAL Id: inria-00074857 \\ https://hal.inria.fr/inria-00074857}

Submitted on 24 May 2006

HAL is a multi-disciplinary open access archive for the deposit and dissemination of scientific research documents, whether they are published or not. The documents may come from teaching and research institutions in France or abroad, or from public or private research centers.
L'archive ouverte pluridisciplinaire HAL, est destinée au dépôt et à la diffusion de documents scientifiques de niveau recherche, publiés ou non, émanant des établissements d'enseignement et de recherche français ou étrangers, des laboratoires publics ou privés. 


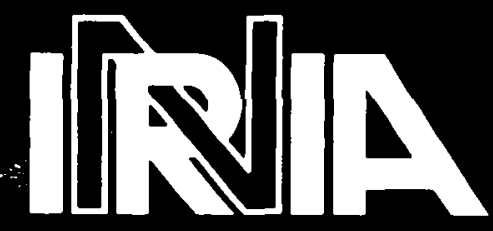

UNITÉ DE RECHERCHE INRIA-ROCQUENCOURT

\section{Rapports de Recherche}

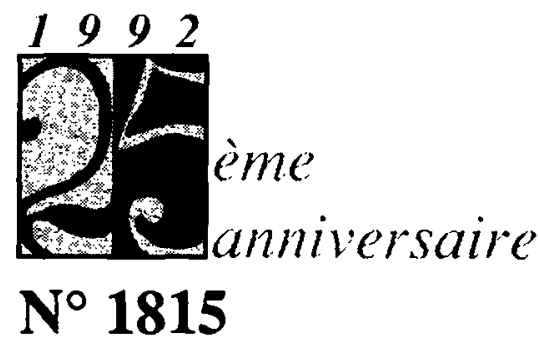

Programme 2

Calcul Symbolique, Programmation et Génie logiciel

\section{STABILITY CONDITIONS FOR SOME DISTRIBUTED SYSTEMS : BUFFERED RANDOM ACCESS SYSTEMS}

Institut National de Recherche en Informatique et en Automatique

Domaine de Voluceau Rocquencourt B.P.105

78153 Le Chesnay Cedex France

Wojciech SZPANKOWSKI

Tél.:(1)39635511 


\title{
STABILITY CONDITIONS FOR SOME DISTRIBUTED SYSTEMS: BUFFERED RANDOM ACCESS SYSTEMS
}

\author{
November 16, 1992 \\ Wojciech Szpankowski* \\ Department of Computer Science \\ Purdue University \\ W. Lafayette, IN 47907 \\ U.S.A.
}

\begin{abstract}
We first consider the standard slotted ALOHA system with a finite number of buffered users. Stability analysis of such a system was initiated in 1979 by Tsybakov and Mikhailov. Since then several bounds on the stability region have been established, however, the exact stability region is known only for the symmetric system and two users ALOHA. This paper proves necessary and sufficient conditions for stability of the ALOHA system, hence solves the problem posed by Tsybakov and Mikhailov. We accomplish this by means of a novel technique based on three simple observations: isolating single queue from the system, applying Loynes' stability criteria for such an isolated queue, and using stochastic dominance and mathematical induction to verify the required stationarity assumptions in the Loynes' criterion. We also point out that our technique can be used to assess stability regions for other multidimensional systems. We illustrate it by deriving the stability region for a buffered system with conflict resolution algorithms. In another paper, Georgiadis and Szpankowski (1992) used this technique to establish stability criteria for the token passing ring system.
\end{abstract}

\section{CONDITIONS DE STABILITE POUR SYSTEMES DISTRIBUES: SYSTEMES A ACCES ALEATOIRE AVEC BUFFERS}

\section{Résumé}

Nous considerons en premier lieu le système ALOHA slotté avec un nombre fini d'utilisateurs munis de buffers. Tsybakov et Mikhailov ont commencé la première analyse de la stabilité d'un tel système en 1979. Depuis de nombreuses bornes de la zone de stabilité ont été mises en évidence, alors que la zone de stabilité a été exactement établies pour seulement deux utilisateurs. Ce papier prouve des conditions nécessaires et suffisantes pour la stabilité du système ALOHA, refermant par là le problème ouvert. par Tsybakov et Mikhailov. Nous utilisons une technique nouvelle basée sur les trois considérations suivantes: isoler un utilisateur unique dans le système, appliquer le critère de Loynes sur cette file d'attente isolée, et utiliser le principe de la récurrence pour e'tendre la condition de stabilité au système complet. Nous mettons en évidence le fait que notre technique peut être utiliser pour de'finir les zones de stabilité pour d'autres problèmes multidimensionnels. Nous illustrons ce propos par l'établisement de la zone de stabilité d'un algorithme de résolution de collision dans un système avec buffers. Dans un autre papier Georgiadis et Szpankowski (1992) ont utilisé cette pour établir des critères de stabilité pour le système de l'anneau a' jeton.

- This research was supported in part by the NSF grants NCR-9206315, CCR-9201078, and INT-8912631, by AFOSR grant 90-0107, and in part by NATO Collahorative Grant 0057/89. This paper was completed while the author was visiting INRIA, Rocquencourt, France, and he wishes to thank INRIA (projects ALGO, MEVAL and REFLECS) for a generous support. 


\section{INTRODUCTION}

A fundamental issue in the design of any distriluuted system is its stability, loosely defined as its ability to possess required properties in the presence of some disturbances. Hereafter, by stability we understand an ability of a system to keep a quantity of interest (e.g., queue length, waiting time, etc.) in a bounded region, or more precisely the existence of the limiting distribution for a quantity of interest.

In this paper, we investigate the buffered ALOHA system, propose a method of evaluating its stability, and show that this new approach can be extended to a larger class of distributed systems. Stability analysis of the buffered ALOHA system was initiated by Tsybakov and Mikhailov (1979) who obtained a simple bound for the stability region, and exact sufficient and necessary conditions for the ergodicity of the symmetric system (e.g., all input rates and probability of transmissions are the same). It was generalized in Szpankowski (1988) who derived some improved bounds for the stability region, and some new bounds for the instability region. Fallin (1981) used the Lyapunov test function approach (cf. Tweedie (1976)) to obtain another bound for the stability region (cf. also Szpankowski (1988)). Recently, Rao and Ephremides (1989), using the stochastic dominance method constructed the best up-todate bound for the stability region for not-too-asymmetric buffered ALOHA system. Finally, Anantharam (1991) - for a simple model of the arrival process - obtained the envelope of the ergodicity region (i.e., a stability region for which there exists a vector of transmission probabilities that assures the system stability).

The exact stability region for the ALOHA model is known only for $M=2$ users system, and the symmetric model. The case $M=2$ was solved by Tsybakov and Mikhailov (1979) who applied general stability criteria for two-dimensional homogeneous Markov chains derived by Malyshev in his seminal paper Malyshev (1972). These general stability criteria have been extended to higher dimensions by Mensikov (1974), and Malyshev and Mensikov (1981). For two-dimensional homogeneous Markov chains Vaninskii and Lazareva (1988), Fayolle (1989) and Rosenkrantz (1989) relaxed some of Malyshev's restrictions (e.g., boundness of the arrival process). It should be said, however, that the above criteria for higher than two-dimensional Markov chains are rather difficult to apply in practice. Despite the fact that these criteria are known for almost twenty years, very few real systems have been analyzed through this approach (see Karatzoglu and Ephremides (1989) for an application).

In this paper, we solve the stability problem of the ALOHA system originally posed by Tsybakov and Mikhailov (1979), that is, we provide exact stability region by establishing necessary and sufficient condition for stability of the ALOHA system. Our approach is novel, and it was already outlined in Szpankowski (1990). This technique was recently rigorized in Georgiadis and Szpankowski (1992) where sufficient and necessary stability condition was 
established for the token passing ring.

Our technique is based on three simple observations. Informally speaking: (i) we show that stability of an $M$-dimensional multiqueue system can be reduced to stability of an isolated single queue; (ii) we apply an old result of Loynes (1962) that allows to assess stability of a general $G|G| 1$ queue with stationary arrival and service processes; (iii) finally, to verify a technical stationarity requirement in Loynes' criteria we apply the stochastic dominance technique and mathematical induction. It should be stressed, however, that within this general framework every multidimensional model requires subtle but significant modifications that are often far from obvious.

This paper is organized as follows. In the next section we present sufficient and necessary conditions for stability of the buffered ALOHA system. Using them, we derived explicit formulas for the stability regions in the case of $M=2$ and $M=3$. The proof of our main result is delayed till Section 3 that also presents a detailed description of our approach. The last section shows how the proposed technique can be extended to other multiqueue systems such as buffered systems with conflict resolıtion algorithms (cf. Capetanakis (1979), Szpankowski (1987), Paterakis et al. (1987)).

\section{MAIN RESULTS}

This section provicles sufficient and necessary stability conditions for the buffered ALOHA system. The proof of our main Theorem 1 is presented in the next section.

\subsection{Model Formulation}

We start with a short description of the buffered ALOHA system (cf. Tsybakov and Mikhailov (1979), Szpankowski (1986)). The system consists of $M$ distributed users, each having an infinite buffer for storing fixed-length packets. The channel is slotted, and a slot duration is equal to a packet transmission time. The arrival process is i.i.d. with respect to slots, and arrival processes are independent from a user to a user. Each nonempty user transmits a packet with probability $r_{i}$ in a slot, where $i \in \mathcal{M}$ and $\mathcal{M}=\{1,2, \ldots, M\}$ is the set of users. If two or more users transmit simultaneously, then a collision occurs and the packets must be retransmitted in future. When exactly one packet is transmitted in a slot, then a successful transmission takes place, the packet is removed from its queue, and another packet, if the queue is nonempty, gets its chance to be served. For technical reasons - and to make our presentation uniform - we additionally introduce the availability of a slot. We assume that a slot is available to all users with probability $1-b$, and blocked with probability $b$. In the standard ALOHA we have $b=0$.

Let $N_{j}^{t}$ represent the queue length in the $j$ th user at the beginning of the $t$ th slot, where $t=0,1, \ldots$ Under the above assumptions, the $M$-dimensional process $\mathrm{N}^{t}=\left(N_{1}^{t}, N_{2}^{t}, \ldots, N_{M}^{t}\right)$ 
is a Markov chuin (cf. Tsybakov and Mikhailov (1979), Szpankowski (1986)) since

$$
N_{j}^{t+1}=\left(N_{j}^{t}-Y_{j}^{t}\right)^{+}+X_{j}^{t}
$$

where $X_{j}^{t}$ represents the number of customers arriving during the $t$ th slot to the $j$ th user with $E X_{j}^{t}=\lambda_{j}<\infty$, and

$$
Y_{j}^{t}=\left(1-B^{t}\right) R_{j}^{t}\left(1-\sum_{k \in \mathcal{M}-\{j\}} R_{k}^{t} \chi\left(N_{k}^{t}\right)\right)^{+} .
$$

In the above: $1-\operatorname{Pr}\left\{R_{j}^{t}=0\right\}=\operatorname{Pr}\left\{R_{j}^{t}=1\right\}=\tau_{j}$, and $\operatorname{Pr}\left\{B^{t}=0\right\}=1-\operatorname{Pr}\left\{B^{t}=1\right\}=1-b$, and for all $k \in \mathbb{N}_{0}$

$$
\chi(k)= \begin{cases}0 & \text { if } k=0 \\ 1 & \text { if } k \geq 1\end{cases}
$$

where $N_{0}$ is a set of nonnegative integers.

\subsection{Stability Criteria}

A multidimensional processes $\mathrm{N}^{t}=\left(N_{1}^{t}, \ldots, N_{M}^{t}\right)$ (not necessarily Markovian process) is stable if for $\mathrm{x} \in \mathbb{N}_{0}^{M}$ the following holds

$$
\lim _{t \rightarrow \infty} \operatorname{Pr}\left\{\mathrm{N}^{t}<\mathbf{x}\right\}=F(\mathbf{x}) \quad \text { and } \quad \lim _{\mathbf{x} \rightarrow \infty} F(\mathbf{x})=1
$$

where $F(\mathbf{x})$ is the limiting distribution function, and by $\mathbf{x} \rightarrow \infty$ we understand that $x_{j} \rightarrow \infty$ for all $j \in \mathcal{M}=\{1, \ldots, M\}$. If a weaker condition holds, namely,

$$
\lim _{\mathbf{x} \rightarrow \infty} \lim _{t \rightarrow \infty} \text { inf } \operatorname{Pr}\left\{\mathbf{N}^{t}<\mathbf{x}\right\}=1
$$

then the process is called sulstable by Loynes (1962), tight by Breiman (1968), and bounded in probability by Meyn and Tweedie (1992). Otherwise, the system is unstable (for more details see Loynes (1962), Borovkov (1976), Walrand (1988)).

We start with an informal overview of our approach. First of all, we construct a modified ALOHA system as follows. Let $\mathcal{P}=(\mathcal{S}, \mathcal{U})$ be a partition of $\mathcal{M}$ such that users in $\mathcal{S} \neq \mathcal{M}$ work exactly in the same manner as in the original ALOHA model, while users in $\mathcal{U}$ persistently attempt to send packets even if their buffers are empty (e.g., dummy packets). We call users in $\mathcal{U}$ persistent (or jamming), and users in $\mathcal{S}$ nonpersistent. Note that the system consisting of users restricted to $\mathcal{S}$ form a smaller copy of the original ALOHA system with a new probability of slot availability.

For a given partition $\mathcal{P}=(\mathcal{S}, \mathcal{U})$ of $\mathcal{M}$ such that $\mathcal{S} \neq \mathcal{M}$, let $\overrightarrow{\mathbf{N}}_{\mathcal{P}}^{t}=\left(\overrightarrow{\mathbf{N}}_{\mathcal{S}}^{t}, \overrightarrow{\mathbf{N}}_{\mathcal{U}}^{t}\right)$ denote the queue lengths in the modified system where $\overline{\mathbf{N}}_{\mathcal{S}}^{t}$ (resp. $\overline{\mathbf{N}}_{\mathcal{U}}^{t}$ ) represents the queue lengths in 
$\mathcal{S}$ (resp. in $\mathcal{U}$ ). Clearly, $\overline{\mathrm{N}}_{\mathcal{p}}^{t}$ dominates the original queueing process $\mathrm{N}^{t}$ (cf. Tsybakov and Mikhailov (1979) and Szpankowski (1986)), that is,

$$
\mathrm{N}^{t} \leq_{s t} \overline{\mathrm{N}}_{\mathcal{P}}^{t}
$$

provided $\mathbf{N}^{0}=\overline{\mathbf{N}}_{\mathcal{P}}^{0}$.

Note that by our construction, the process $\overline{\mathbf{N}}_{\mathcal{S}}^{t}$ is an $|\mathcal{S}|$-dimensional Markov chain that mimics the behavior of the ALOHA system. Since $|\mathcal{S}|<M$, we can apply mathematical induction to establish its stability. We further assume that the Markov chain $\overline{\mathbf{N}}_{\mathcal{S}}^{t}$ is stationary and ergodic (this will be verified later in Section 3 ). We denote this stationary version as $\overline{\mathbf{N}}_{\mathcal{S}}^{0}$ to indicate that the process starts from the stationary distribution.

Let $Y_{j}^{t}(\mathcal{S})$ be the output process from the $j$ th queue in the dominant system. We write $P_{\text {succ }}^{j}(\mathcal{S})=E Y_{j}^{t}(\mathcal{S})$ to denote the probability of a successful transmission from the $j$ th user in the dominant system provided $Y_{j}^{t}(\mathcal{S})$ is a stationary sequence. This is assumed to hold as a simple consequence of our assumption regarding stationarity of $\overline{\mathbf{N}}_{\mathcal{s}}^{t}$. We set $1-b=$ $\prod_{k \in \mathcal{U}-\{j\}}\left(1-r_{k}\right)$ for $j \in \mathcal{M}$, and then (cf. Tsybakov and Mikhailov (1979), Szpankowski (1986))

$$
P_{s u c c}^{j}(S)=r_{j} \prod_{k \in \mathcal{U}-\{j\}}\left(1-r_{k}\right) \sum_{\mathbf{z}_{\mathcal{S}} \in \Theta_{\mathcal{S}}}\left(\operatorname{Pr}\left\{\chi\left(\overline{\mathbf{N}}_{\mathcal{S}}^{0}\right)=\mathbf{z}_{\mathcal{S}}\right\} \prod_{k \in \mathcal{S}-\{j\}}\left(1-r_{k}\right)^{z_{k}}\right),
$$

where the summation is over the set.

$$
\Theta_{\mathcal{S}}=\left\{\mathbf{z}_{\mathcal{S}}: \mathbf{z}_{\mathcal{S}}=\left(z_{1}, \ldots, z_{|\mathcal{S}|}\right), z_{j} \in\{0,1\}, j \in \mathcal{S}\right\} .
$$

In the above, $\chi\left(\overline{\mathrm{N}}_{\mathcal{S}}^{0}\right)=\left(\chi\left(\bar{N}_{i_{1}}^{0}\right), \ldots, \chi\left(\bar{N}_{i_{|S|}}\right)\right)$ where $i_{j} \in \mathcal{S}$ for all $j=1, \ldots,|\mathcal{S}|$, and by $k \in \mathcal{A}-\{j\}$ we mean that $k$ belongs to $\mathcal{A}$ and it is not equal to $j$ (even if $j \notin \mathcal{A}$ ).

The next theorem is our main finding, and it provides sufficient and necessary condition for stability of the buffered ALOHA system. It is proved in Section 3. In the below theorem, we write iff* to mean: if and only if with possible exception of boundary points of a stability region. (In fact, our technique usually does not establish stability of boundary points, however, mostly only minor modifications are necessary to prove instability of boundary points; e.g., Georgiadis and Szpankowski (1992)).

Theorem 1. The buffered ALOHA system is stable iff $\lambda \in \mathcal{R}$ with

$$
\mathcal{R}=\bigcup_{k=1}^{M}\left\{\lambda=\left(\lambda_{1}, \ldots, \lambda_{M}\right): \lambda_{j}<P_{s u c c}^{j}\left(\mathcal{M}_{k}\right), \text { for all } j \in \mathcal{M}\right\}
$$

where $\mathcal{M}_{k}=\mathcal{M}-\{k\}$ and $P_{\text {succ }}^{j}\left(\mathcal{M}_{k}\right)$ is defined in (2.0) with $\mathcal{S}=\mathcal{M}_{k}$. 
Remarks. (i) Note that according to Theorem 1 only partitions $\mathcal{P}_{k}=\left(\mathcal{M}_{k},\{k\}\right)$ contribute to the stability region $\mathcal{R}$. In fact, in Section 3 we prove that $\bigcup_{k=1}^{M} \mathcal{R}_{\mathcal{M}_{k}}=\bigcup_{\mathcal{S} \subset \mathcal{M}} \mathcal{R}_{\mathcal{S}}$ where $\mathcal{R}_{\mathcal{S}}$ is the stability region for the partition $(\mathcal{S}, \mathcal{U})$.

(ii) The proof presented in Section 3 allows to establish local stability conditions, that is, stability of a subset $\mathcal{S}$ of all queues $\mathcal{M}$ (in this case, we assume that the remaining queues $\mathcal{M}-\mathcal{S}$ are unstable). The local stability region $\mathcal{R}_{\mathcal{S}}$ is given by formula (2.8) with $\mathcal{M}_{k}$ replaced by $\mathcal{S}_{k}=\xi-\{k\}$.

(iii) Our proof of Theorem 1 can be used to give a detailed characterization of the instability region. More precisely, we can partition the instability region $\overline{\mathcal{R}}$ into subregions $\overline{\mathcal{R}}\left(k_{1}, \ldots, k_{d}\right)$ $\left(k_{i} \in \mathcal{M}\right.$ for $\left.i=1, \ldots, d\right)$ such that for $\lambda \in \overline{\mathcal{R}}\left(k_{1}, \ldots, k_{d}\right)$ only queues $N_{k_{1}}^{t}, \ldots, N_{k_{d}}^{t}$ are unstable while the remaining are stable. For example, in Theorem 7 of Section 3 we show that in the region

$$
\overline{\mathcal{R}}(k)=\left\{\lambda=\left(\lambda_{1}, \ldots, \lambda_{M}\right): \lambda_{k}>P_{s u c c}^{k}\left(\mathcal{M}_{k}\right) \text { and } \lambda_{j}<P_{s u c c}^{j}\left(\mathcal{M}_{k}\right) \text { for all } j \in \mathcal{M}-\{k\}\right\}
$$

only the $k$ th queue is unstable while the others are stable.

(iv) The stability region for the ALOHA system (and several others) can be simplified if one considers the so called envelope of the stability regions which is defined as the set of all $\lambda=\left(\lambda_{1}, \ldots, \lambda_{M}\right)$ such that there exists a vector of transmission probabilities $\left(r_{1}, \ldots, r_{M}\right)$ for which the ALOHA system is stable. Tsybakov and Mikhailov (1979) conjectured that the envelope $\mathcal{E}$ of the stability region for the ALOHA can be characterized as follows

$$
\mathcal{E}=\left\{\lambda=\left(\lambda_{1}, \ldots, \lambda_{M}\right): \lambda_{j}<r_{j} \prod_{k \neq j}\left(1-r_{k}\right), 0 \leq r_{j} \leq 1 \text { for all } j \in \mathcal{M}\right\} .
$$

This result was proved for a simple input process by Anantharam (1991). However, for general ALOHA it is still an open problem. We believe that our Theorem 1 can be used to settle this conjecture, but we do not tackled this in the current paper.

\subsection{Special Cases and Bounds}

Provided Theorem 1 is proved, we can apply it to establish stability regions for $M=2$ and $M=3$, and the symmetric ALOHA system. We also discuss some bounds on $\mathcal{R}$. Stability characterization for $M=3$ is new.

We start with $M=2$, and let $\mathcal{P}_{1}=\left(\mathcal{M}_{1},\{1\}\right)$ and $\mathcal{P}_{2}=\left(\mathcal{M}_{2},\{2\}\right)$ where $\mathcal{M}_{1}=\{2\}$ and $\mathcal{M}_{2}=\{1\}$. We write $\mathcal{R}_{i}$ to denote the stability region for the partition $\mathcal{P}_{i}$ for $i=1,2$. Below, only the construction of $\mathcal{R}_{1}$ is discussed in details. By (2.6) we have

$$
\begin{aligned}
& P_{\text {succ }}^{1}\left(\mathcal{M}_{1}\right)=r_{1}\left(\operatorname{Pr}\left\{\bar{N}_{2}^{0}=0\right\}+\left(1-r_{2}\right) \operatorname{Pr}\left\{\bar{N}_{2}^{0} \geq 1\right\}\right) \\
& P_{\text {succ }}^{2}\left(\mathcal{M}_{1}\right)=r_{2}\left(1-r_{1}\right) .
\end{aligned}
$$



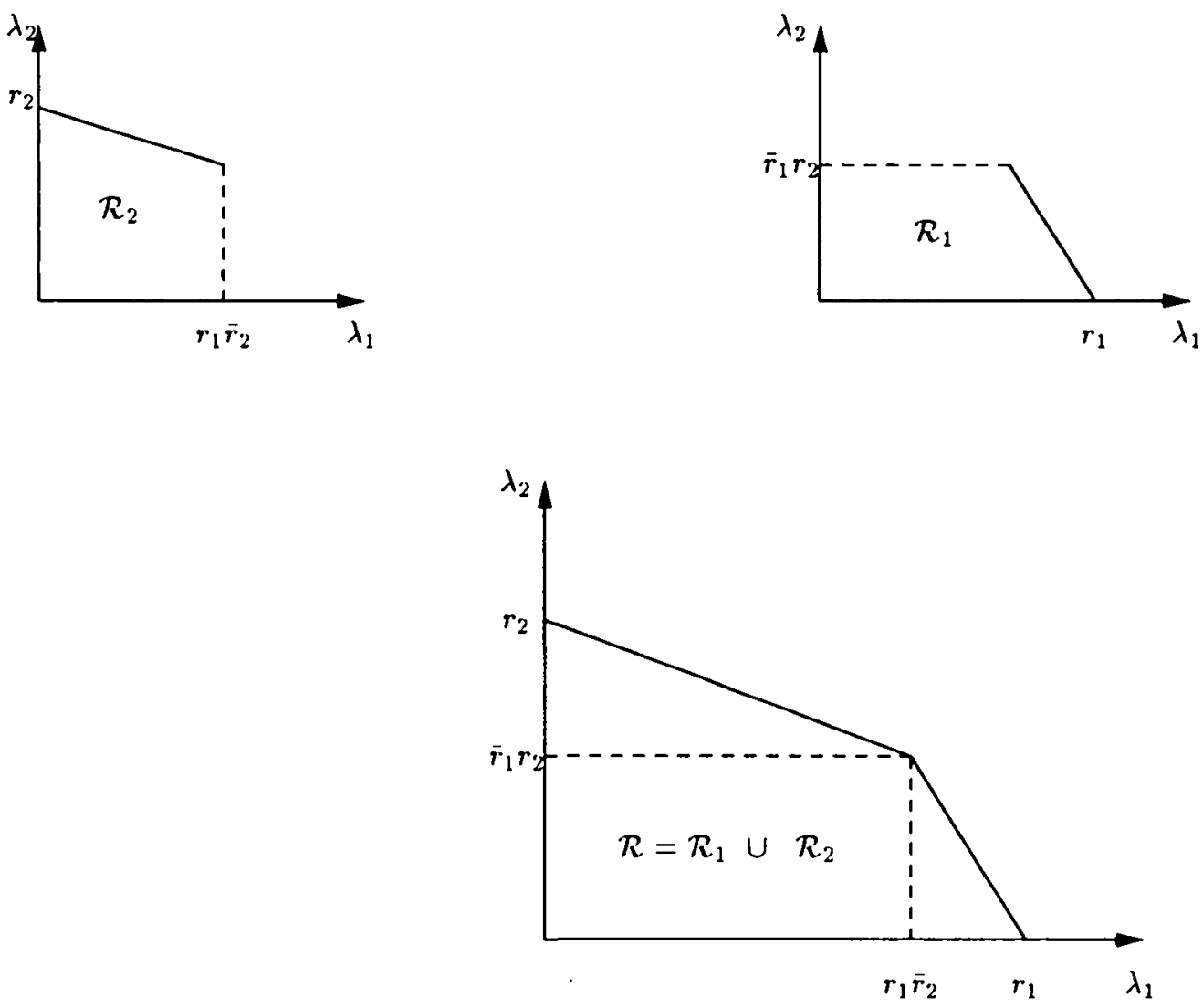

Figure 1: Stability region $\mathcal{R}$ for $M=2$ as a union of stability regions $\mathcal{R}_{1}$ and $\mathcal{R}_{2}$.

But, for $\lambda_{2}<P_{\text {succ }}^{2}\left(\mathcal{M}_{1}\right)$ the probability $\operatorname{Pr}\left\{\bar{N}_{2}^{0} \geq 1\right\}=\lambda_{2} /\left(r_{2}\left(1-r_{1}\right)\right)$, and hence one obtains the stability region $\mathcal{R}_{1}$. In a similar manner, considering $\mathcal{M}_{2}$ we obtain the stability region $\mathcal{R}_{2}$. The whole stability region $\mathcal{R}$ is the sum of $\mathcal{R}_{1}$ and $\mathcal{R}_{2}$ and it is illustrated in Figure 1.

Putting everything together, we summarize the stability result for $M=2$ in the following corollary. Under stronger assumptions, this result was already known to Tsybakov and Mikhailov (1979) (cf. also Rao and Ephremides (1989)).

Corollary 2. For $M=2$, the buffered $A L O H A$ system is stable for all $\left(\lambda_{1}, \lambda_{2}\right) \in \mathcal{R}$ such that

$$
\mathcal{R}=\left\{\lambda_{1}<r_{1}\left(1-\lambda_{2} / \bar{r}_{1}\right) \text { and } \lambda_{2}<\bar{r}_{1} r_{2}\right\} \cup\left\{\lambda_{1}<r_{1} \bar{r}_{2} \text { and } \lambda_{2}<r_{2}\left(1-\lambda_{1} / \bar{r}_{2}\right)\right\}
$$

and the system is unstable for $\left(\lambda_{1}, \lambda_{2}\right) \in \overline{\mathcal{R}}$ where

$$
\overline{\mathcal{R}}=\left\{\lambda_{1}>r_{1}\left(1-\lambda_{2} / \bar{r}_{1}\right) \text { or } \lambda_{2}>\bar{r}_{1} r_{2}\right\} \cap\left\{\lambda_{1}>r_{1} \bar{r}_{2} \text { or } \lambda_{2}>r_{2}\left(1-\lambda_{1} / \bar{r}_{2}\right)\right\},
$$

where $\bar{r}_{j}=1-r_{j}$.

Now, we consider the case $M=3$ which is more intricate. We investigate three partitions $\mathcal{P}_{i}=\left(\mathcal{M}_{i},\{i\}\right)$ where $\mathcal{M}_{1}=\{2,3\}, \mathcal{M}_{2}=\{1,3\}$ and $\mathcal{M}_{3}=\{1,2\}$. Only the first partition 
will be discussed in details. As before, the stability region $\mathcal{R}$ is the sum of three regions $\mathcal{R}_{1}$, $\mathcal{R}_{2}$ and $\mathcal{R}_{3}$ each corresponding to $\mathcal{M}_{1}, \mathcal{M}_{2}$ and $\mathcal{M}_{3}$, respectively.

We now consider $\mathcal{R}_{1}$. The dominant system can be viewed as a two-user ALOHA system with appropriate probability for the slot availability. Such a system was analyzed by Nain (1985). We first briefly summarize some of Nain's results adopted to our setting.

Let $F_{1}(x, y)$ denote the generating function of $\left(N_{2}^{t}, N_{3}^{t}\right)$ with slot availability $1-b=1-r_{1}$. Then, with a minor modification, it is proved in Nain (1985) (see also Szpankowski (1986)) that

$$
\begin{aligned}
& \lambda_{2}=\bar{r}_{1} r_{2} \bar{r}_{3}\left(1-F_{1}(0,1)\right)+\bar{r}_{1} r_{2} r_{3}\left(F_{1}(1,0)-F_{1}(0,0)\right), \\
& \lambda_{3}=\bar{r}_{1} \bar{r}_{2} r_{3}\left(1-F_{1}(1,0)\right)+\bar{r}_{1} r_{2} r_{3}\left(F_{1}(0,1)-F_{1}(0,0)\right),
\end{aligned}
$$

where, as before, $\bar{r}_{i}=1-r_{i}$. Moreover, for $z_{2}, z_{3} \in\{0,1\}$ we use the following simplified notation $P_{1}\left(z_{2}, z_{3}\right)=\operatorname{Pr}\left\{\chi\left(\bar{N}_{2}\right)=z_{2}, \chi\left(\bar{N}_{3}\right)=z_{3}\right\}$ with the first user being a persistent one. From Nain (1985) we have

$$
P_{1}(0,0)=\left(1-\frac{\lambda_{2}}{\bar{r}_{3} \bar{r}_{1}}-\frac{\lambda_{3}}{r_{3} \bar{r}_{1}}\right) \exp \left(\frac{\gamma(1)}{2 \pi i}\right) \int_{|t|=1} \frac{\log g(t)}{t(t-\gamma(1))} d t
$$

or

$$
P_{1}(0,0)=\left(1-\frac{\lambda_{2}}{r_{2} \bar{r}_{1}}-\frac{\lambda_{3}}{\bar{r}_{2} \bar{r}_{1}}\right) \exp \left(\frac{\gamma(1)}{2 \pi i}\right) \int_{|t|=1} \frac{\log g_{1}(t)}{t(t-\gamma(1))} d t
$$

depending whether $P_{1}(0,0)$ is computed from $F_{1}(0, y)$ or $F_{1}(x, 0)$. We note that $\gamma(1)$ and $g(t)$ depend of the input rates $\lambda_{2}$ and $\lambda_{3}$ ! For example, $F_{1}(1,0)$ corresponding to $(2.11 \mathrm{a})$ becomes

$$
\begin{aligned}
F_{1}(1,0) & =\left(1-\frac{\lambda_{2}}{\bar{r}_{3} \bar{r}_{1}}-\frac{\lambda_{3}}{r_{3} \bar{r}_{1}}\right)\left(\frac{1-r_{3} \bar{r}_{1}}{1-r_{3} \bar{r}_{1}-r_{2} \bar{r}_{1}}\right) \int_{|t|=1} \frac{\log g(t)}{\left((t-\gamma(1))^{2}\right.} d t \\
& -\frac{r_{2} \bar{r}_{1}}{1-r_{2} \bar{r}_{1}-r_{3} \bar{r}_{1}} P_{1}(0,0)
\end{aligned}
$$

The region of validity of (2.11) is defined in Nain (1985). In $(2.11),\left.\gamma(x)\right|_{x=1}$ is the inverse of a conformal mapping of a unit circle onto a curve $L_{x}$ defined in Nain (1985) (p. 54 and Lemma 4.1). The functions $g(t)$ and $g_{1}(t)$ are defined in Nain (1985), too.

Now we are ready to present the stability region for $M=3$ ALOHA system. By (2.6) for $\mathcal{P}_{1}$ we have

$$
\begin{gathered}
P_{\text {succ }}^{1}\left(\mathcal{M}_{1}\right)=r_{1}\left(P_{1}(0,0)+P_{1}(1,0)\left(1-r_{2}\right)+P_{1}(0,1)\left(1-r_{3}\right)+P_{1}(1,1)\left(1-r_{2}\right)\left(1-r_{3}\right)\right), \\
P_{\text {succ }}^{2}\left(\mathcal{M}_{1}\right)=r_{2}\left(1-r_{1}\right)\left(1-\left(1-F_{1}(1,0)\right) r_{3}\right), \\
P_{\text {succ }}^{3}\left(\mathcal{M}_{1}\right)=r_{3}\left(1-r_{1}\right)\left(1-\left(1-F_{1}(0,1)\right) r_{2}\right),
\end{gathered}
$$

where the probabilities $P_{1}\left(z_{2}, z_{3}\right)$ must be computed according to (2.10)-(2.12). For example, using (2.10) we can show that

$$
P_{\text {succ }}^{1}\left(\mathcal{M}_{1}\right)=r_{1}\left\{1-\frac{\lambda_{2} \bar{r}_{2} / \bar{r}_{1}+\lambda_{3} \bar{r}_{3} / \bar{r}_{1}+r_{2} r_{3}\left(P_{1}(0,0)-1\right)}{1-r_{2}-r_{3}}\right\} .
$$




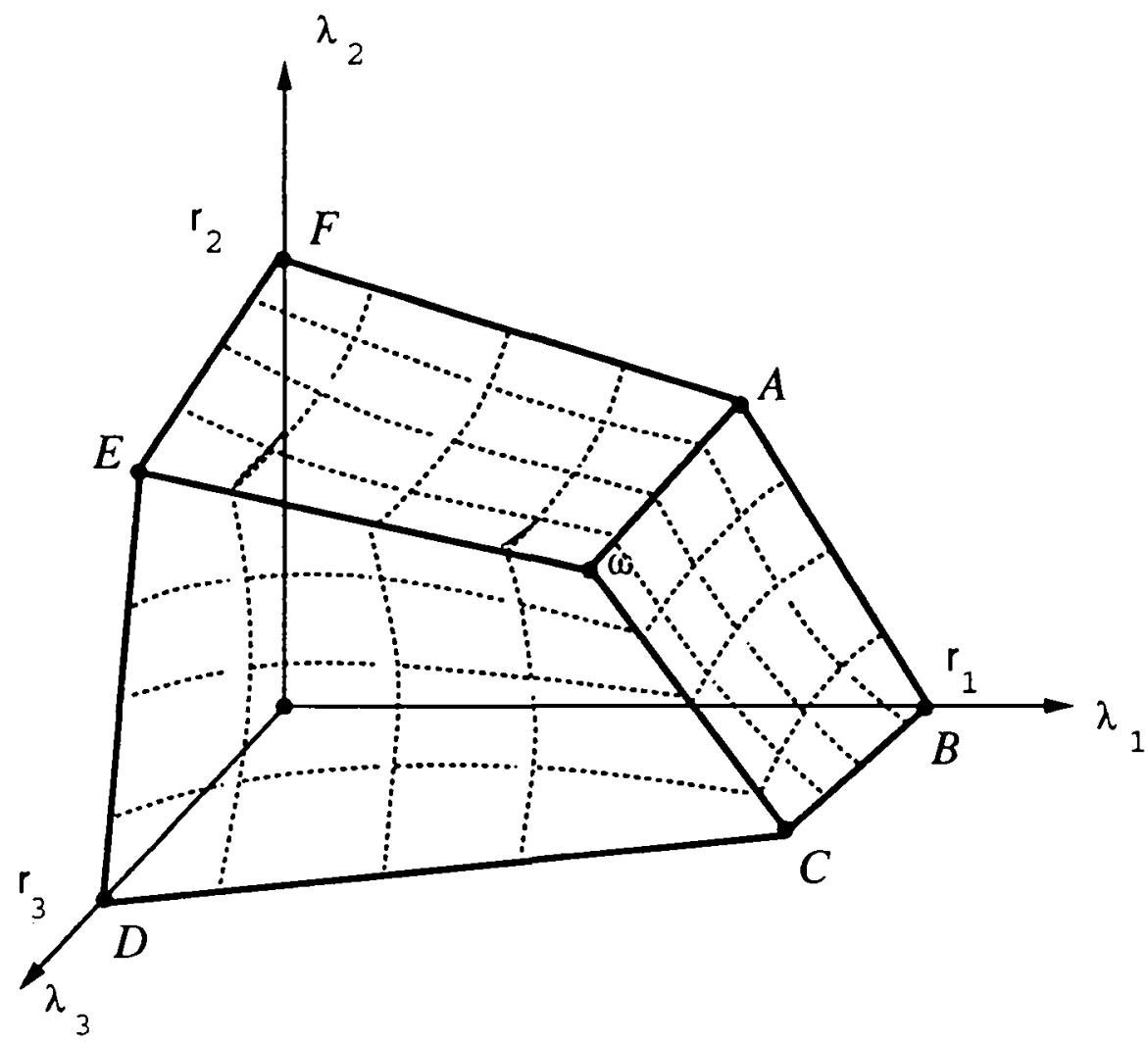

Figure 2: Stability region for $M=3$ users in the slotted ALOHA system.

In a similar manner, we can express $P_{\text {succ }}^{2}\left(\mathcal{M}_{1}\right)$ and $P_{\text {succ }}^{3}\left(\mathcal{M}_{1}\right)$ in terms of $P_{1}(0,0)$.

In summary, we obtain the following corollary.

Corollary 3. The buffered ALOHA system is stable iff $\left(\lambda_{1}, \lambda_{2}, \lambda_{3}\right) \in R=\bigcup_{i=1}^{3} \mathcal{R}_{i}$ where

$$
\begin{aligned}
& \mathcal{R}_{1}=\left\{\lambda_{1}<P_{\text {succ }}^{1}\left(\mathcal{M}_{1}\right), \lambda_{2}<r_{2} \bar{r}_{1}\left(1-\left(1-F_{1}(1,0)\right) r_{3}\right), \lambda_{3}<r_{3} \bar{r}_{1}\left(1-\left(1-F_{1}(0,1)\right) r_{2}\right)\right\} \\
& \mathcal{R}_{2}=\left\{\lambda_{1}<r_{1} \bar{r}_{2}\left(1-\left(1-F_{2}(1,0)\right) r_{3}\right), \lambda_{2}<P_{s u c c}^{2}\left(\mathcal{M}_{2}\right), \lambda_{3}<r_{3} \bar{r}_{2}\left(1-\left(1-F_{2}(0,1)\right) r_{1}\right)\right\} \\
& \mathcal{R}_{3}=\left\{\lambda_{2}<r_{1} \bar{r}_{3}\left(1-\left(1-F_{3}(1,0)\right) r_{2}\right), \lambda_{2}<r_{2} \bar{r}_{3}\left(1-\left(1-F_{3}(0,1)\right) r_{1}\right), \lambda_{3}<P_{\text {succ }}^{3}\left(\mathcal{M}_{3}\right)\right\}
\end{aligned}
$$

where the appropriate probabilities above are computed from the Nain's (1985) model as discussed above (cf. (2.10)-(2.12)) with some obvious modifications.

Stability region $\mathcal{R}$ for $M=3$ is shown in our Figure 2. Note that the following points belong to the boundary of the stability region: $\omega=\left(\lambda_{1}, \lambda_{2}, \lambda_{3}\right)=\left(r_{1} \bar{r}_{2} \bar{r}_{3}, \bar{r}_{1} r_{2} \bar{r}_{3}, \bar{r}_{1} \bar{r}_{2} r_{3}\right)$, $A=\left(r_{1} \bar{r}_{2}, \bar{r}_{1} r_{2}, 0\right)$, and $B=\left(r_{1}, 0,0\right), C=\left(r_{1} \bar{r}_{3}, 0, \bar{r}_{1} r_{3}\right), D=\left(0,0, r_{3}\right), E=\left(0, r_{2} \bar{r}_{3}, \bar{r}_{2} r_{3}\right)$ and $F=\left(0, r_{2}, 0\right)$. In passing, we stress the fact that the probability of success $P_{\text {succ }}^{j}\left(\mathcal{M}_{k}\right)$ does depend explicitly on the probability $P_{1}(0,0)$, which is a nonlinear function of the input rates. This implies that the boundaries of the stability region for the ALOHA system for $M \geq 3$ are not linear functions of $\left(\lambda_{1}, \lambda_{2}, \lambda_{3}\right)$. 
A generalization of the above to $M>3$ is even more challenging, since we need to estimate the probability of empty/nonempty buffers in three and higher dimensional ALOHA systems. Nevertheless for $M>3$, some bounds are easy to obtain from Theorem 1. For example, the bounds derived by Tsybakov and Mikhailov (1979) (cf. also Szpankowski (1988)) directly follow from (2.6) and (2.8). Indeed, since $\prod_{k \in \mathcal{M},}\left(1-r_{k}\right)^{z_{k}} \geq \prod_{k \in \mathcal{M}}\left(1-r_{k}\right)$, one immediately proves from (2.6) that $\lambda_{j}<r_{j} \prod_{k \in \mathcal{M},}\left(1-r_{k}\right)$ for $j \in \mathcal{M}$ is sufficient for stability of the ALOHA system. On the other hand, since $\prod_{k \in \mathcal{M}}\left(1-r_{k}\right)^{z_{k}} \leq 1$ we prove that $\lambda \geq r_{j}$ for some $j \in \mathcal{M}$, is sufficient for instability of the ALOHA system.

The above simple bounds can be used to establish sufficient and necessary conditions for stability of the symmetric ALOHA systern. We prove the following result which was already known to Tsybakov and Mikhailov (1979)

Corollary 4. Let $r_{j}=r$ and $\lambda_{j}=\lambda$ for all $j \in \mathcal{M}$. Then, such a symmetric ALOHA system is stable if and only if the following holds

$$
\lambda<r(1-r)^{M-1} .
$$

Proof. This directly follows from Theorem 1 since in the symmetric case we really deal with a one dimensional problem. The stability region in this case is the intersection of the line $\lambda_{1}=\lambda_{2}=\cdots=\lambda_{M}=\lambda$ with the region $\mathcal{R}$ as defined in (2.8) for $r_{1}=r_{2}=\cdots=r_{M}=r$. Even simpler proof can be obtained, by noting that (2.14) is a direct consequence of our upper bound $\lambda_{j}<r_{j} \prod_{k \in \mathcal{M}}\left(1-r_{k}\right)$ for $j \in \mathcal{M}$ just derived (2.8). Setting in this inequality the symmetric model assumptions, we obtain (2.14). From Tsybakov and Mikhailov (1979), (cf. Szpankowski (1986)) we also know that (2.14) is necessary for the stability.

To obtain more sophisticated bounds for the stability region $\mathcal{R}$, one needs a tighter estimate for the probability $\operatorname{Pr}\left\{\chi\left(\overline{\mathbf{N}}_{s}^{t}\right)=z_{\mathcal{S}}\right\}$ in (2.6) (cf. Szpankowski (1988), and Rao and Ephremides (1989)). For example, in Rao and Ephremides (1989) all probabilities $\operatorname{Pr}\left\{\chi\left(\overline{\mathbf{N}}_{\mathcal{S}}^{t}\right)=z_{\mathcal{S}}\right\}$ in (2.6) are skillfully bounded by a one dimensional probability $\operatorname{Pr}\left\{\vec{N}_{k}^{t} \geq 1\right\}$ for $k \in \mathcal{S}$.

\section{ANALYSIS: AN ALTERNATIVE APPROACH}

In this section, we present our approach to compute stability conditions for multiqueue systems. We concentrate on proving Theorem 1, however, generality of the approach should be apparent from our discussion. We first prove sufficient conditions (cf. Section 3.1), and then necessary conditions (cf. Section 3.2) for stability. In the proof below, we shall use two general results, namely the so called isolation lemma (cf. Lemma 5) and Loynes' scheme Loynes (1962) (cf. Borovkov (1976)) adopted to our situation (cf. Lemma 6).

We start with the isolation lemma which states that for substability of a multidimensional 
process $\mathbf{N}^{t}$ (not necessary Markovian) one requires substability of its components. More precisely, we prove the following.

Lemma 5. (i) If for all $j \in \mathcal{M}$ the one dimensional processes $N_{j}^{t}$ are substable, then the $M$-dimensional process $\mathrm{N}^{t}=\left(N_{1}^{t}, N_{2}^{t}, \ldots, N_{M}^{t}\right)$ is substable.

(ii) If for some $j$, say $j^{*}, N_{j *}^{t}$ is unstable, then $\mathrm{N}^{t}$ is also unstable.

(iii) If $\mathrm{N}^{t}$ is a Markov chain defined on a countable state space, then the stability of $N_{j}^{t}$ for all $j \in \mathcal{M}$ implies stability of the multidimensional Markov chain $\mathbf{N}^{t}$.

Proof. We first prove part (i). By definition (2.4), for all $j \in \mathcal{M}$ we have $\lim _{x_{j}, t \rightarrow \infty} \sup \operatorname{Pr}\left\{N_{j}^{t}>\right.$ $\left.x_{j}\right\}=0$. But,

$$
\begin{aligned}
1 \geq \lim _{x \rightarrow \infty} \liminf _{n \rightarrow \infty} \operatorname{Pr}\left\{N_{j}^{n} \leq x_{j}, \text { for } \quad j\right. & =1,2, \ldots, M\} \\
\geq & 1-\sum_{j=1}^{M} \lim _{x_{j} \rightarrow \infty} \limsup _{n \rightarrow \infty} \operatorname{Pr}\left\{N_{j}^{n}>x_{j}\right\}=1 .
\end{aligned}
$$

Thus, $\lim _{x \rightarrow \infty} \lim _{t \rightarrow \infty}$ inf $\operatorname{Pr}\left\{\mathbf{N}^{t}<\mathbf{x}\right\}=1$, as needed. If $\mathrm{N}^{t}$ is a Markov chain defined on a countable state space, then substability implies stability since such a Markov chain always converges to a random variable, which might be dishonest. This proves (iii). For results concerning Markov chains defined on more general spaces the reader is referred to Meyn and Tweedie (1992).

For part (ii) we notice that instability of $N_{j *}^{t}$ implies $\lim _{x_{j} \rightarrow \infty} \lim _{t \rightarrow \infty} \inf \operatorname{Pr}\left\{N_{j *}^{t}<x_{j *}\right\}<$ 1. Hence,

$$
\lim _{x_{j \bullet} \rightarrow \infty} \lim _{t \rightarrow \infty} \inf \operatorname{Pr}\left\{\mathbf{N}^{t}<\mathbf{x}\right\} \leq \lim _{x, \rightarrow \infty} \lim _{t \rightarrow \infty} \inf \operatorname{Pr}\left\{N_{j *}^{t}<x_{j_{*}}\right\}<1
$$

which proves Lemma 5.

Our second result is a simple consequence of the Loynes' scheme Loynes (1962) (cf. Borovkov (1976)). It provides stability criteria for a general single queue described by the following stochastic equation

$$
N_{j}^{t+1}=\left(N_{j}^{t}-Y_{j}^{t}\right)^{+}+X_{j}^{t}
$$

with stationary input sequences.

Lemma 6. [Loynes 1962), Borovkov (1976)]. Let the pair $\left(X_{j}^{t}, Y_{j}^{t}\right)$ be a strictly stationary and ergodic process. We denote by $\lambda_{j}=E X_{j}$ and $E Y_{j}$ the corresponding mean values of $X_{j}^{t}$ and $Y_{j}^{t}$ respectively. Then the following holds

(i) if $\lambda_{j}<E Y_{j}$, then the queue length $N_{j}^{t}$ is stable in the sense of definition (2.3), 
(ii) if $\lambda_{j}>E Y_{j}$, then the queue is unstable, and $\lim _{t \rightarrow \infty}, N_{j}^{t}=\infty$ (a.s.).

Proof. The proof is a direct consequence of well known results. Indeed, set $W_{j}^{t+1}=N_{j}^{t+1}-X_{j}^{t}$ and $U_{j}^{t}=X_{j}^{t-1}-Y_{j}^{t}$. Then,

$$
W_{j}^{t+1}=\left(W_{j}^{t}+U_{j}^{t}\right)^{+} .
$$

and, by Loynes (1962) and Borovkov (1976) $N_{j}^{t}$ is stable for $E U_{j}^{t}<0$ and unstable for $E U_{j}^{t}>0$. To prove the second part of (ii), we note that $W_{j}^{t+1} \geq W_{j}^{0}+\sum_{k=0}^{t} U_{j}^{t}$, and by Birkhoff's Individual Ergodic Theorcm (Breiman (1968)) $\sum_{k=0}^{t} U_{j}^{t} \rightarrow \infty$ (a.s.) provided $E U_{j}^{t}>0$.

\subsection{Sufficient Conditions}

We use mathematical induction to establish sufficient condition for stability of the Markov chain $\mathrm{N}^{t}$ describing the ALOHA system. This part of the proof resembles the idea already used by Georgiadis and Szpankowski (1992).

For $M=1$ the proof is easy. It suffices to note that the average drift becomes $E\left\{N^{t+1}-\right.$ $\left.N^{t} \mid N^{t} \geq 1\right\}=\lambda-E Y^{t}$. Hence, the proof follows from the Lyapunov function method (cf. Tweedie (1976), Szpankowski (1990)).

Now we assume that Theorem 1 is true for $M-1$ and we prove that it can be extended to $M$ queues. The main idea is to consider a modified ALOHA system in which the set of users $\mathcal{M}$ is partition into $\mathcal{P}=(\mathcal{S}, \mathcal{U})$ with $\mathcal{S} \neq \mathcal{M}$ where $\mathcal{S}$ is a copy of the ALOHA model that mimics its behavior, while users in $\mathcal{U}$ persistently jam users in $\mathcal{S}$ (i.e., a user in $\mathcal{U}$ attempts to send a dummy packet even if empty).

The modified system dominates stochastically the original one, as stated in (2.5). Hence, we concentrate on proving stability condition for the dominant system represented by $\overline{\mathbf{N}}_{\mathcal{P}}^{t}=$ $\left(\overrightarrow{\mathbf{N}}_{\mathcal{S}}^{t}, \overline{\mathbf{N}}_{\mathcal{U}}^{t}\right)$. We first consider users in $\mathcal{S}$, and establish stability condition for $\overline{\mathbf{N}}_{\mathcal{S}}^{t}$. Note that the set of users restricted to $\mathcal{S}$ is a smaller copy of the ALOHA model, with the probability of slot availability equal to $1-b=\prod_{k \in \mathcal{U}-\{j\}}\left(1-r_{k}\right)$. Hence, the probability of success $P_{s u c c}^{j}(\mathcal{S})$ is given by (2.6). Let $\lambda_{S}=\left(\lambda_{i_{1}}, \ldots, \lambda_{i_{|S|} \mid}\right)$ where $i_{j} \in \mathcal{S}$ for $1 \leq i_{j} \leq|\mathcal{S}|$. Since $|\mathcal{S}|<M$, by mathematical induction arguments $\overline{\mathbf{N}}_{\mathcal{S}}^{t}$ is stable according to Theorem 1 for $\lambda_{\mathcal{S}} \in \mathcal{R}_{\mathcal{S}}^{1}$ where

$$
\mathcal{R}_{\mathcal{S}}^{1}=\bigcup_{\ell=1}^{|\mathcal{S}|}\left\{\lambda_{\mathcal{S}}: \lambda_{j}<P_{\text {succ }}^{j}\left(\mathcal{S}_{\ell}\right) \text { for all } j \in \mathcal{S}\right\},
$$

and $S_{\ell}=\mathcal{S}-\{\ell\}$.

We now provide stability condition for a persistent queue $j \in \mathcal{U}$. The idea is to apply Lemma 6 to an isolated persistent queue $j \in \mathcal{U}$. For this we need to establish stationarity and ergodicity of the output process $Y_{j}^{t}(\mathcal{S})$.

We proceed as follows. Let $\lambda_{\mathcal{S}} \in \mathcal{R}_{\mathcal{S}}^{1}$ (cf. (3.2)), and consider a persistent user, say $j \in \mathcal{U}$. The Markov chain $\overline{\mathrm{N}}_{\mathcal{S}}^{t}$ is stable by the mathematical induction. More precisely, this Markov 
chain is ergodic, hence there exists a unique honest stationary distribution $\pi$ for this process. But, $\left(\overline{\mathrm{N}}_{\mathcal{S}}^{t}, Y_{j}^{t+1}(\mathcal{S})\right)$ is another $|\mathcal{S}|+1$-dimensional Markov chain. It is easy to see that this chain is irreducible and aperiodic, too. Moreover, $Y_{j}^{t}(S)$ is bounded from the above by one, hence $\left(\overrightarrow{\mathrm{N}}_{\mathcal{S}}^{t}, Y_{j}^{t+1}(\mathcal{S})\right)$ is substable. By Lemma 5 (iii), this implies that the $|\mathcal{S}|+1$-dimensional Markov chain is stable, and also ergodic. Let its stationary distribution be $\bar{\pi}$.

Now we construct a stationary version of the process $\left(\overline{\mathrm{N}}_{\mathcal{S}}^{t}, Y_{j}^{t+1}(\mathcal{S})\right)$ by starting it with the initial distribution $\bar{\pi}$. This will imply that the process $Y_{j}^{t}(\mathcal{S})$ for $j \in \mathcal{U}$ is stationary and ergodic for such an initial distribution. Then, by Lemma 6 the $j$ th persistent queue is stable if $\lambda_{j}<E Y_{j}^{t}(\mathcal{S})=P_{s u c c}^{j}(\mathcal{S})$ where $P_{s u c c}^{j}(\mathcal{S})$ is given by (2.6). This is true for every persistent queue $j \in \mathcal{U}$, and hence by Lemma 5 the process $\overline{\mathbf{N}}_{\mathcal{U}}^{t}$ is stable for $\lambda \in \mathcal{R}_{\mathcal{S}}^{2}$ where

$$
\mathcal{R}_{\mathcal{S}}^{2}=\left\{\lambda_{\mathcal{U}}: \lambda_{j}<P_{\text {succ }}^{j}(\mathcal{S}) \text { for all } j \in \mathcal{U}\right\},
$$

and the Markov chain $\overline{\mathbf{N}}_{\mathcal{P}}^{t}=\left(\overline{\mathbf{N}}_{\mathcal{S}}^{t}, \overline{\mathbf{N}}_{\mathcal{U}}^{t}\right)$ is stable for $\lambda \in \mathcal{R}_{\mathcal{S}}^{\prime}=\mathcal{R}_{\mathcal{S}}^{1} \cup \mathcal{R}_{\mathcal{S}}^{2}$.

So far, we have established stability condition for a given partition $\mathcal{P}=(\mathcal{S}, \mathcal{U})$ of $\mathcal{M}$. Clearly, the dominant system represented by $\overline{\mathbf{N}}^{t}$ is stable if there exists a partition $\mathcal{P}$ such that $\overline{\mathbf{N}}_{\mathcal{P}}^{t}$ is stable. Therefore, the stability region for $\overline{\mathrm{N}}^{t}$ becomes $\mathcal{R}^{\prime}=\bigcup_{\mathcal{S} \subset \mathcal{M}} \mathcal{R}_{\mathcal{S}}^{\prime}$ where the sum is over all subsets of $\mathcal{M}$ such that $\mathcal{S} \neq \mathcal{M}$.

We prove now that $\mathcal{R}^{\prime}=\mathcal{R}$ where $\mathcal{R}$ is defined in (2.8) of Theorem 1, that is, $\mathcal{R}=\bigcup_{k=1}^{M} \mathcal{R}_{\mathcal{M}_{k}}$ where $\mathcal{R}_{\mathcal{M}_{k}}=\left\{\lambda: \lambda_{j}<P_{\text {succ }}^{j}\left(\mathcal{M}_{k}\right), j \in \mathcal{M}\right\}$. First, however, we simplify the expression for $\mathcal{R}^{\prime}$. Note that $\bigcup_{\mathcal{S} \subset \mathcal{M}} \mathcal{R}_{\mathcal{S}}^{\prime}=\bigcup_{k=1}^{M} \mathcal{R}_{\mathcal{M}_{k}}^{\prime}$ where by (3.2) and (3.3)

$$
\mathcal{R}_{\mathcal{M}_{k}}^{\prime}=\bigcup_{\substack{\ell=1 \\ \ell \neq k}}^{M}\left\{\lambda: \lambda_{j}<P_{s u c c}^{j}\left(\mathcal{M}_{k, \ell}\right) \text { for all } j \in \mathcal{M}-\{k\} \text { and } \lambda_{k}<P_{s u c c}^{k}\left(\mathcal{M}_{k}\right)\right\}
$$

with $\mathcal{M}_{k, \ell}=\mathcal{M}-\{k, l\}$. This shows that the only partitions that contribute to the ultimate stability region are of the form $\left(\mathcal{M}_{k},\{k\}\right)$. Indeed, it is a simple consequence of $\left(1-r_{k}\right)^{z_{k}} \geq$ $\left(1-r_{k}\right)$ where $z_{k} \in\{0,1\}$, and the following monotonicity property of the probability of success $P_{\text {succ }}^{j}(\mathcal{S})$ (cf. Tsybakov and Mikhailov (1979), and Szpankowski (1986))

$$
\mathcal{S}^{\prime} \subset \mathcal{S} \quad \Longrightarrow \quad P_{\text {succ }}^{j}\left(\mathcal{S}^{\prime}\right) \leq P_{\text {succ }}^{j}(\mathcal{S})
$$

We now prove $\mathcal{R}_{\mathcal{M}_{k}}=\mathcal{R}_{\mathcal{M}_{k}}^{\prime}$ which suffices for $\mathcal{R}=\mathcal{R}^{\prime}$. Clearly, $P_{\text {succ }}^{j}\left(\mathcal{M}_{k, \ell}\right) \leq P_{\text {succ }}^{j}\left(\mathcal{M}_{j, \ell}\right)$ (cf. Tsybakov and Mikhailov (1979), Szpankowski (1986) and (1988)). Indeed, this is true since under the partition $\left(\mathcal{M}_{k, \ell},\{k, \ell\}\right)$ the $k$ th user is never empty, hence its queue length can be made always larger that the the queue length at user $k$ under the partition $\left(\mathcal{M}_{j, \ell},\{j, \ell\}\right)$. Therefore,

$$
\mathcal{R}_{\mathcal{M}_{k}}^{\prime}=\left\{\lambda: \lambda_{j}<P_{s u c c}^{j}\left(\mathcal{M}_{k, j}\right) \text { for all } j \in \mathcal{M}-\{k\} \text { and } \lambda_{k}<P_{s u c c}^{k}\left(\mathcal{M}_{k}\right)\right\}
$$


Now it. suffices to show that $P_{\text {succ }}^{j}\left(\mathcal{M}_{k, j}\right)=P_{\text {succ }}^{j}\left(\mathcal{M}_{k}\right)$. But this is easy. Consider the definition (2.6) of the probalility of success, and proceed as follows

$$
\begin{aligned}
P_{\text {succ }}^{j}\left(\mathcal{M}_{k, j}\right) & =r_{j}\left(1-r_{k}\right) \sum_{\mathbf{z}_{\mathcal{M}_{k}, j} \in \Theta_{\mathcal{M}_{k, j}}} \operatorname{Pr}\left\{\mathbf{N}_{\mathcal{M}_{k, j}}=\mathbf{z}_{\mathcal{M}_{k, j}}\right\} \prod_{\ell \in \mathcal{M}_{k}}\left(1-r_{\ell}\right)^{z_{\ell}} \\
& =r_{j}\left(1-r_{k}\right) \sum_{\mathbf{z}_{\mathcal{M}_{k}} \in \Theta_{\mathcal{M}_{k}}} \operatorname{Pr}\left\{\mathbf{N}_{\mathcal{M}_{k}}=\mathbf{z}_{\mathcal{M}_{k}}\right\} \prod_{\ell \in \mathcal{M}_{k}}\left(1-r_{\ell}\right)^{z_{\ell}}\left(\operatorname{Pr}\left\{N_{j}=0\right\}+\operatorname{Pr}\left\{N_{j} \geq 1\right\}\right) \\
& =P_{\text {succ }}^{j}\left(\mathcal{M}_{k}\right)
\end{aligned}
$$

This shows that $\mathcal{R}=\mathcal{R}^{\prime}$, and completes the sufficient part of the proof for Theorem 1 .

\subsection{Necessary Conditions}

In this section we prove necessary conditions for stability of the ALOHA system. More precisely, we establish the following result, which directly implies the "necessary part" of Theorem 1.

Theorem 7. If $\lambda \in \overline{\mathcal{R}}=\bigcap_{k=1}^{M} \overline{\mathcal{R}}_{k}$ where

$\overline{\mathcal{R}}_{k}=\left\{\lambda=\left(\lambda_{1}, \ldots, \lambda_{M}\right): \lambda_{k}>P_{\text {succ }}^{k}\left(\mathcal{M}_{k}\right)\right.$ and $\lambda_{j}>P_{\text {succ }}^{j}\left(\mathcal{M}_{k}\right)$ for some $\left.j \in \mathcal{M}-\{k\}\right\}$,

then the Markov chain $\mathbf{N}^{t}$ is unstable. In particular, for $\lambda \in \bar{R}_{k}$ the kth queue $N_{k}^{t} \rightarrow \infty$ with positive probability.

We carry out the proof of Theorem 7 in three steps. The main idea behind the proof is to show that:

(i) if (3.6) holds, then the $k$ th queue under the partition $\left(\mathcal{M}_{k},\{k\}\right)$ is unstable in the dominant system $\overline{\mathbf{N}}_{\mathcal{P}}^{t}$,

(ii) the last assertion can be extended to the original system (i.e., still only for queue $k$ );

(iii) we can generalize (ii) above to other queues in the original system.

Step 1. Instability of the dominant system

Let us consider the dominant system under the partition $\mathcal{P}=\left(\mathcal{M}_{k},\{k\}\right)$. Define a subset $\overline{\mathcal{R}}_{k}(k)$ of the instability region $\overline{\mathcal{R}}_{k}$ as

$\overline{\mathcal{R}}_{k}(k)=\left\{\lambda=\left(\lambda_{1}, \ldots, \lambda_{M}\right): \lambda_{k}>P_{s u c c}^{k}\left(\mathcal{M}_{k}\right)\right.$ and $\lambda_{j}<P_{s u c c}^{j}\left(\mathcal{M}_{k}\right)$ for all $\left.j \in \mathcal{M}-\{k\}\right\}$.

The arrival process to a persistent queue (e.g., the $k$ th one) does not effect the stability condition for nonpersistent queues represented by the Markov chain $\overline{\mathbf{N}}_{\mathcal{S}}^{t}$. Hence, for $\lambda \in \overline{\mathcal{R}}_{k}(k)$ 
the Markov chain $\overline{\mathbf{N}}_{\mathcal{S}}^{t}$ is stable, as proved in Section 3.1. Using the same construction as above, we can assure stationarity and ergodicity of $\overline{\mathbf{N}}_{\mathcal{S}}^{t}$ as well as the output process $Y_{k}^{t}\left(\mathcal{M}_{k}\right)$. Therefore, the stationarity and ergodicity assumptions of Loynes' scheme hold, and we can apply Lemma 6 to the $k$ th queue. In particular, part (ii) implies that if $\lambda_{k}>E Y_{k}^{t}\left(\mathcal{M}_{k}\right)=$ $P_{s u c c}^{k}\left(\mathcal{M}_{k}\right)$, then $\bar{N}_{k}^{t} \rightarrow \infty$ (a.s.).

\section{Step 2. Instability of the $k$ th queue in the original system}

We now prove that for $\lambda \in \overline{\mathcal{R}}_{k}(k)$ the $k$ th queue is also unstable in the original system. We shall use a coupling argument to show that with positive probability the dominant system and the original system are indistinguishable for $\lambda \in \overline{\mathcal{R}}_{k}(k)$.

Consider the dominant system $\overline{\mathbf{N}}_{\mathcal{P}}^{t}$ where $\mathcal{P}=\left(\mathcal{M}_{k},\{k\}\right)$. From Step 1, we know that for $\lambda \in \overline{\mathcal{R}}_{k}(k)$ the $k$ th queue length in the dominant system tends to infinity (a.s.). Let $\overline{N_{k}^{0}}=d>0$. Then, there exists a set of positive probability such that the sample paths of $\bar{N}_{k}^{t}$ never returns to zero. Let now $\overline{N_{k}^{0}}=N_{k}^{0}=d$, so on this set of positive probability, the dominant system and the original system are identical, that is, $\mathbf{N}^{t}=\overline{\mathbf{N}}_{\mathcal{P}}^{t}$ for all $t \geq 0$. This implies that the original system never returns to zero on these sample paths. In summary, with positive probability the $k$ th queue in the original system tends to infinity.

Step 3. Extension of instability to the whole set $\overline{\mathcal{R}}$.

We shall prove now that if $\lambda \in \overline{\mathcal{R}}_{k}$, where $\overline{\mathcal{R}}_{k}$ is defined in (3.6), then the original system, hence the Markov chain $\mathbf{N}^{t}$, is unstable.

Consider another queue, say $\ell \neq k$ in the original system. Consider first only the region $\overline{\mathcal{R}}_{k}(k)$, and increase the the input rate $\lambda_{\ell}$ (beyond $P_{s u c c}^{\ell}\left(\mathcal{M}_{k}\right)$ ). It is known (cf. Tsybakov and Mikhailov (1979), and Szpankowski (1986)) that such an increase will only lead to the increase of the $k$ th queue length. Therefore, at least for one queue, namely the $k$ th one, we have $N_{k}^{t} \rightarrow \infty$ with positive probability. But, since $\mathrm{N}^{t}$ is a Markov chain, then by Lemma 5 (ii) the whole system is unstable (nonergodic). This completes the proof of Theorem 7 , and establishes our main Theorem 1.

\section{FURTHER RESULTS AND CONCLUSION}

The technique proposed Section 3 is clearly not limited to the ALOHA system. It is, however, restricted to multidimensional queueing systems since the real engine of our proof is the Loynes' scheme which allows for the treatment of non-Markovian subsystems (of persistent queues). Several other multiqueue models can be treated in a similar manner. In Georgiadis and Szpankowski (1992) we used this technique to prove the stability of the token passing ring.

To generalize our scheme, we repeat main ingredients of our proof of Theorem 1 . As the first step, we partition the set of users $\mathcal{M}$ into nonpersistent users $\mathcal{S}$ and persistent ones $\mathcal{U}$. 
It is assumed that users in $\mathcal{S}$ form a smaller copy of the original system, while persistent users in $S$ attempt sending dummy packets even when empty. We denote by $\overline{\mathbf{N}}_{\mathcal{F}}^{t}$ the queueing process representing this modified system. For our technique to work, we need the following two properties:

\section{(P1) Monotonicity Condition}

The queue lengths in all users increase whenever dummy messages are sent by a persistent user, that is $\mathrm{N}^{t} \leq_{s t} \overline{\mathrm{N}}^{t}$ for all $t \geq 0$.

\section{(P2) Stationary Version of $\mathcal{S}$}

The set of users $\mathcal{S}$ is a smaller copy of the original system. We also request that the departure process from $\mathcal{S}$ that effects a nonpersistent queue $k \in \mathcal{U}$ is stationary and ergndic (so Loynes' scheme :an be used for establishing stability of a nonpersistent queue).

If the above two properties are satisfied, then we can carry out our analysis, and establish sufficient and necessary condition for stability. We illustrate it again, on another multiqueue systems (we leave, however, details of the proof to an interested reader who should follow our footsteps from Section 3).

We shall investigate a buffered multiaccess system with conflict resolution algorithm. The system works in a manner similar to ALOHA except that it adopts another multiaccess protocol, namely the so called blocked conflict resolution algorithm (blocked CRA) of Capetanakis (1979), and Tsybakov and Mikhailov (1978).

The system works in the following manner. If there is a collision, then all users not involved in it are blocked, and are not allowed to transmit until the current collision is resolved. The collision is solved by a divide-and-conquer algorithm, that is, all users involved in a collision flip a coin and only those who flipped "tails" are allowed to transmit in the next slot. This process is continued until all users in the current collision are successfully transmitted. The quality of such a systems depends on the length $L^{t}$ of the conflict resolution session (CRS), where $t$ is a nonnegative integer representing the beginning of the $t$ th CRS. More details can be found in Capetanakis (1979), Tsybakov and Mikhailov (1978), and Szpankowski (1987).

It is easy to verify that properties (P1) and (P2) hold for the above system. Reproducing our previous analysis, we can establish the following result.

Theorem 8. The buffered multiaccess system with a blocked conflict resolution algorithm is stable iff $\lambda \in \mathcal{R}$ with

$$
\mathcal{R}=\bigcup_{k=1}^{M}\left\{\lambda=\left(\lambda_{1}, \ldots, \lambda_{M}\right): \lambda_{j} E L\left(\mathcal{M}_{k}\right)<1 \quad \text { for all } j \in \mathcal{M}\right\}
$$


where $E L\left(\mathcal{M}_{k}\right)$ denotes the average session length under the partition $\left(\mathcal{M}_{k},\{k\}\right)$ in the stationary regime.

The following result is a direct consequence of Theorem 8 and elementary properties of the protocol (cf. Capetanakis (1979), Szpankowki (1987)).

Corollary 9. The buffered CRA system with $M=2$ users is stable if and only if $\left(\lambda_{1}, \lambda_{2}\right) \in \mathcal{R}$ with

$$
\begin{aligned}
\mathcal{R} & =\left\{\lambda_{1}<1 / \ell_{2}=0.2 \text { and } \lambda_{2}<1-4 \lambda_{1}\right\} \cup\left\{\lambda_{1}<1-4 \lambda_{2} \text { and } \lambda_{2}<1 / \ell_{2}=0.2\right\} \\
& =\left\{\lambda: \lambda_{2}+4 \lambda_{1}<1 \text { or } \lambda_{1}+4 \lambda_{2}<1\right\}
\end{aligned}
$$

where the Markov chain $\mathrm{N}^{t}$ is imbeded at the beginning of CRS.

Furthermore, noting that $E L_{m}<\alpha m+\beta$, where $\alpha=2.8867$ and $\beta=1.2336$ (cf. Capetanakis (979) and Szpankowski (1987)), we obtain the following bound for the stability region.

Corollary 10. The buffered CRA system is stable if $\lambda \in \mathcal{R}_{1}$, where

$$
\mathcal{R}_{1}=\bigcup_{k=1}^{M}\left\{\lambda=\left(\lambda_{1}, \ldots, \lambda_{M}\right): \lambda_{j}<\frac{1-\alpha \sum_{i \neq k} \lambda_{i}}{\alpha+\beta} \quad \text { for all } \quad j \in \mathcal{M}\right\}
$$

where the constants $\alpha$ and $\beta$ are given above.

Finally, it should be mentioned that our technique can be extended to multiply channels (i.e., multiserver case) when several packets are sent simultaneously. This even works for infinite number of servers (cf. Georgiadis and Szpankowski (1992)). For such multidimensional models Malyshev's criteria do not work since homogeneity property is not preserved. It might be interesting to see whether our approach can be used to extend Malyshev's criteria.

\section{ACKNOWLEDGMENT}

It is my pleasure to acknowledge several conversations that I have had with Professor Jean Walrand (University of California at Berkeley) on stability problems. I am particularly obliged to Dr. Leonidas Georgiadis (IBM T.J. Watson Research Division) for very careful reading of previous versions of the paper, numerous enlighting discussions, and several suggestions that led to important improvements of the text and better understanding of stability problems.

I would also like to thank an anonymous referee, and Dr. R. Chang (IBM T.J. Watson Research Division), Yorktown Heights, and Dr. Philippe Jacquet, INRIA, France for several comments that led to improved version of this paper. 


\section{References}

[1] Anantharam, V. (1991), The stability region of the finite-user slotted ALOHA protocol, IEEE Trans. Information Theory, 37, 535-540.

[2] Borovkov, A.A. (1976), Stochastic Processes in Queueing Theory, Springer-Verlag, New York.

[3] Breiman, I. (1968), Probability, Addison-Wesley, Reading, MA.

[4] Capetanakis, J. (1979), Tree Algorithms for Packet broadcast Channels, IEEE Trans. Information Theory, 25, 505-515.

[5] Falin, G. (1981), On ergodicity of a multiaccess system, Tech. Kibern., 4, 126-131.

[6] Fayolle, G.(1989), On random walks arising in queueing systems: Ergodicity and transience via quadratic forms as Lyaponov functions. Part I., Queueing Systems, 5, 167-184.

[7] Georgiadis, L., Szpankowski, W. (1992), Stability of token passing rings, Queueing Systems, 11, 7-33.

[8] M. Karatzoglu and A. Ephremides (1989), Ergodicity of $M$-dimensional Random Walks and Random Access Systems, preprint.

[9] Loynes, R. (1962), The stability of a queue with non-independent inter-arrival and service times, Proc. Camb. Philos. Soc., 58, 497-520.

[10] Malyshev, V.A. (1972), Classification of two-dimensional positive random walks and almost linear semimartingals, Dokl. Akad., Nauk SSSR, 22, 136-138.

[11] Malyshev, V.A. and Mensikov, M.V. (1981), Ergodicity, continuity and analyticity of countable Markov chains, Trans. Moscow Math. Soc., 1-18.

[12] Mensikov, M.V. (1974), Ergodicity and transience conditions for random walks in the positive octant of space, Soviet. Math. Dokl., 15, 1118-1121.

[13] S. Meyn and R.L. Tweedie (1992), Criteria for Stability of Markovian Processes I: Discrete Time Chains, Adv. Appl. Prob., 24, 542-574.

[14] Nain, P. (1985), Analysis of a two-mode ALOHA network with infinite capacity buffer, Proc. of Intern. Seminar on Computer Networking and Performance Evaluation, Tokyo, North-Holland, Amsterdam, 49-64,

[15] Paterakis, M., Georgiadis, L., and Papantoni-Kazakos, P. (1987), On the relationship between the finite and the infinite population models for a class of RAA's, IEEE Trans. Commun., 35, 1239-1240.

[16] Rao, R. and Ephremides, A.(1989), On the stability of interacting queues in multiple access system, IEEE Trans. Inform. Theory 34, 918-930.

[17] Rosenkrantz, W. (1989), Ergodicity conditions for two dimensional Markov chains on the positive quadrant, Probab. Theory and Related Fields, 83, 309-319. 5 
[18] Szpankowski, W. (1986), Bounds for queue lengths in a contention packet broadcast system, IEEE Trans. on Commun., 34, 1132-1140.

[19] Szpankowski, W. (1987), On a recurrence Equation Arising in the Analysis of Conflict Resolution Algorithms, Stochastic Models, 3, 89-114.

[20] Szpankowski, W. (1988), Stability conditions for multi-dimensional queueing systems with computer applications, Oper. Res, 36, 944-957.

[21] Szpankowski, W. (1990), Towards computable stability criteria for some multidimensional stochastic processes, in Stochastic Analysis of Computer and Communication Systems (ed. H. Takagi), Elsevier Science Publications B. V. (North-Holland), 131-172.

[22] Tsybakov, B. and Mikhailov, W. (1978), Free Synchronous Packet Access in a Broadcast Channel with Feedback, Probl. Peredachii Infor., 14, 259-280.

[23] Tsybakov, B. and Mikhailov, W. (1979), Ergodicity of slotted ALOHA system, Probl. Peredachii Infor., 15, 73-87.

[24] Tweedie, R..L. (1976), Criteria for classifying general Markov chains, Adv. Appl. Prob., 8, $737-771$.

[25] Vaninskii, K. and Lazareva, B. (1988), Ergodicity and nonrecurrence conditions of a homogeneous Markov chain in the positive quadrant, Problemy Peredachi Informatsii, 24, 105-110.

[26] Walrand. J. (1988), An Introduction to Queueing Networks, Prentice Hall, New Jersey. 
ISSN 0249-6399 